\title{
The True Catalytically Active Structure in Mo- V Based Mixed Oxide Catalysts for Selective Oxidation of Acrolein
}

Satoshi Ishikawa ${ }^{1 *}$, Yudai Yamada ${ }^{1}$, Naoki Kashio ${ }^{1}$, Nagisa Noda ${ }^{1}$, Kosuke Shimoda

${ }^{1}$, Mio Hayashi ${ }^{1}$, Toru Murayama ${ }^{2}$, Wataru Ueda ${ }^{1 *}$

${ }^{1}$ Department of Material and Life Chemistry, Faculty of Engineering, Kanagawa

University, 3-27-1, Rokkakubashi, Kanagawa-ku, Yokohama 221-8686, Japan

${ }^{2}$ Research Center for Gold Chemistry, Graduate School of Urban Environmental

Sciences, Tokyo Metropolitan University, 1-1 Minami-osawa, Hachioji, Tokyo 192-0397, Japan

\section{Corresponding Author}

*E-mail: sishikawa@ kanagawa-u.ac.jps (S. Ishikawa)

*E-mail: uedaw@kanagawa-u.ac.jp (W. Ueda) 


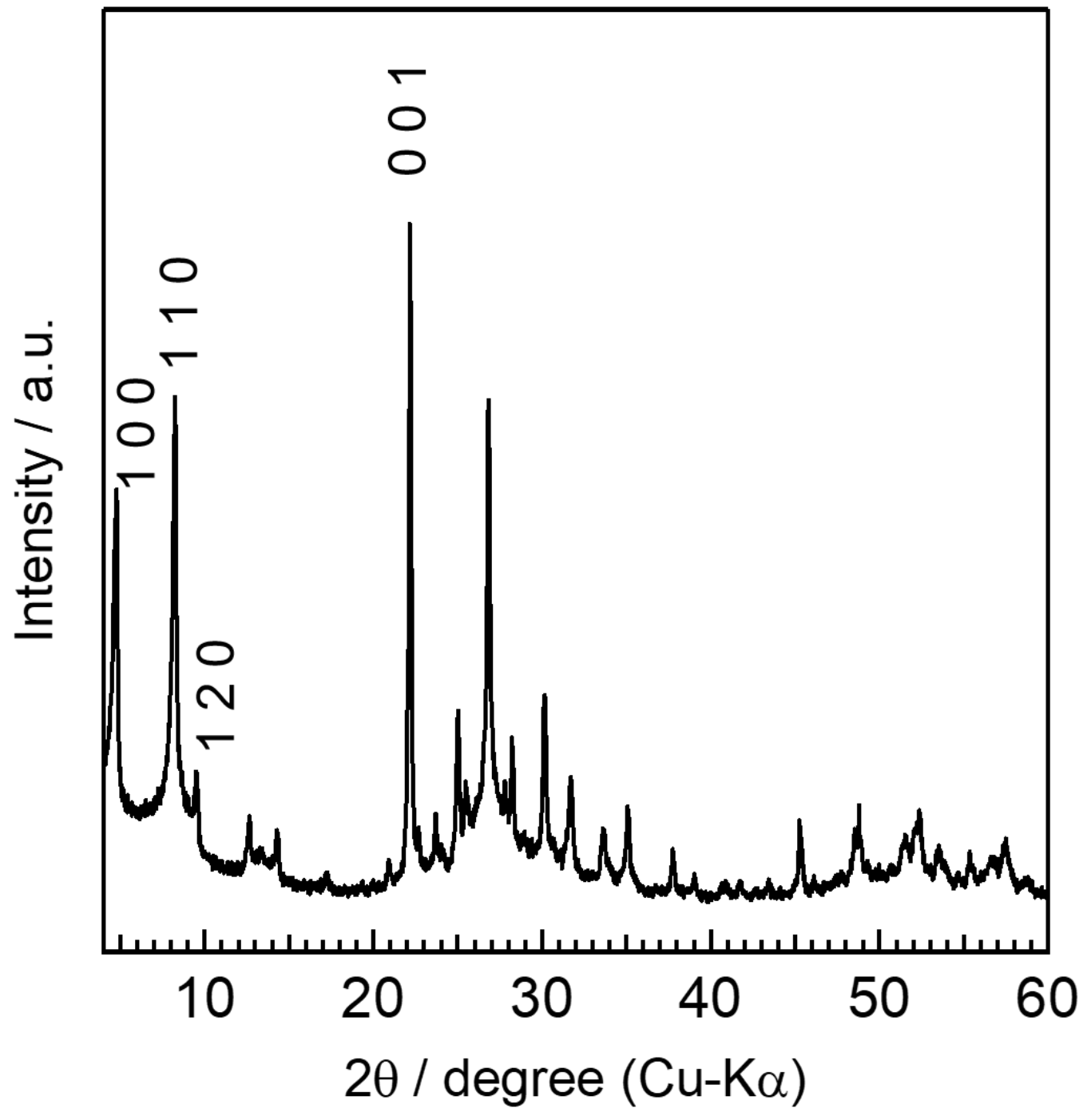

Figure S1. XRD pattern for Tri-MoVO. 

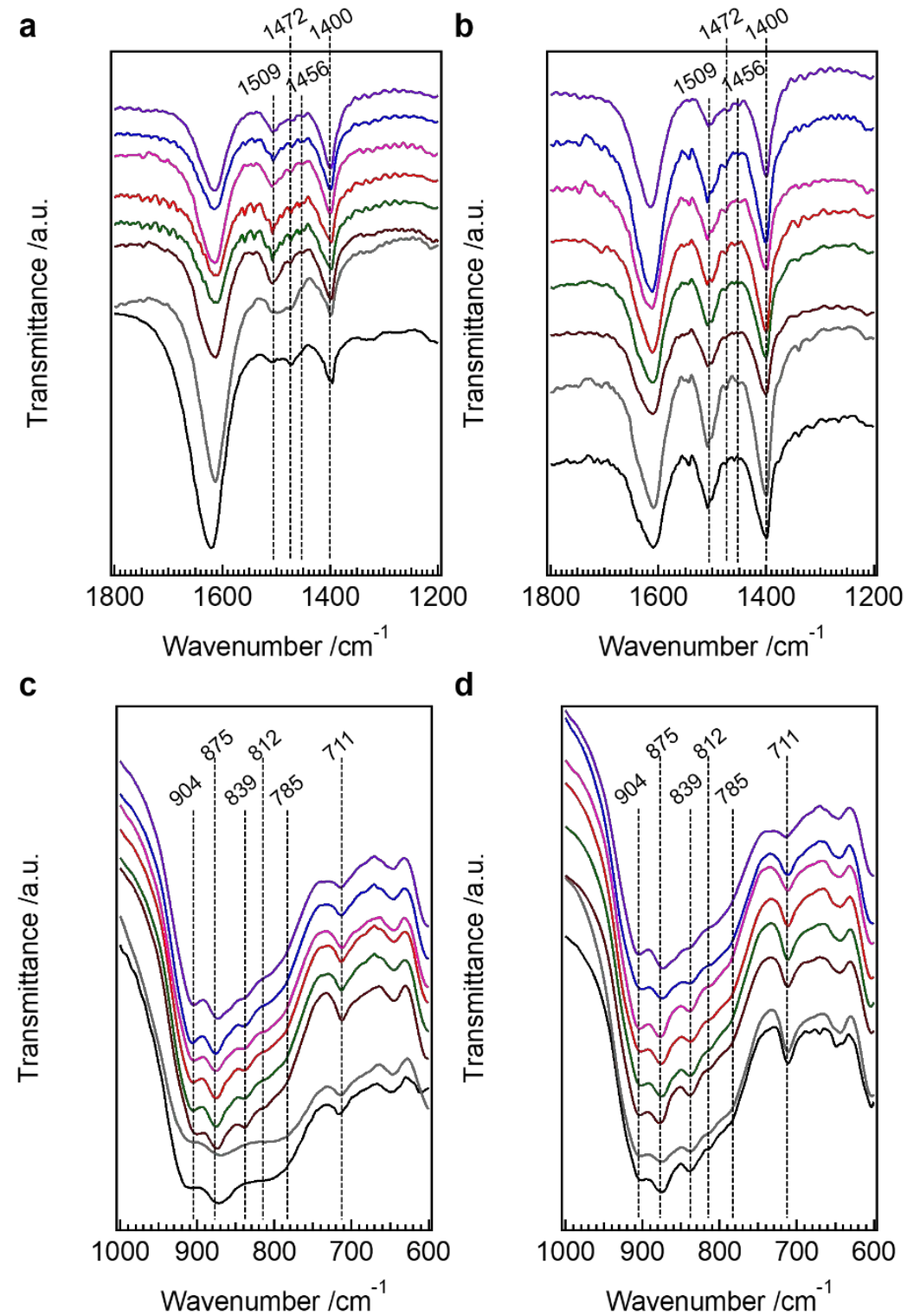

Figure S2. IR spectra of $x \mathrm{~W}$ a, c and $x \mathrm{~W}$-amm b, d. a, b IR spectra in the range of $1200 \sim 1800 \mathrm{~cm}^{-1}$. c, d IR spectra in the range of $600 \sim 1000 \mathrm{~cm}^{-1}$. Black, $0 \mathrm{~W}$ or $0 \mathrm{~W}$-amm; gray, $0.3 \mathrm{~W}$ or $0.3 \mathrm{~W}$-amm; brown, $0.7 \mathrm{~W}$ or $0.5 \mathrm{~W}$-amm; green, $0.8 \mathrm{~W}$ or $0.6 \mathrm{~W}$-amm; red, $1.3 \mathrm{~W}$ or $1.0 \mathrm{~W}$-amm; pink, $1.7 \mathrm{~W}$ or $1.4 \mathrm{~W}$-amm; blue, $1.8 \mathrm{~W}$ or $1.8 \mathrm{~W}$-amm; purple, $2.9 \mathrm{~W}$. 
a

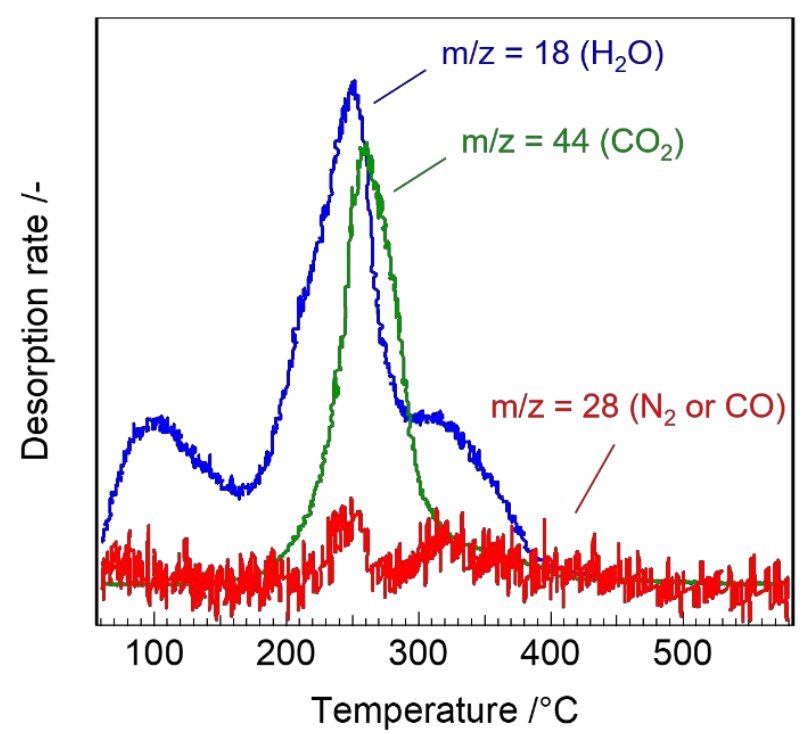

b

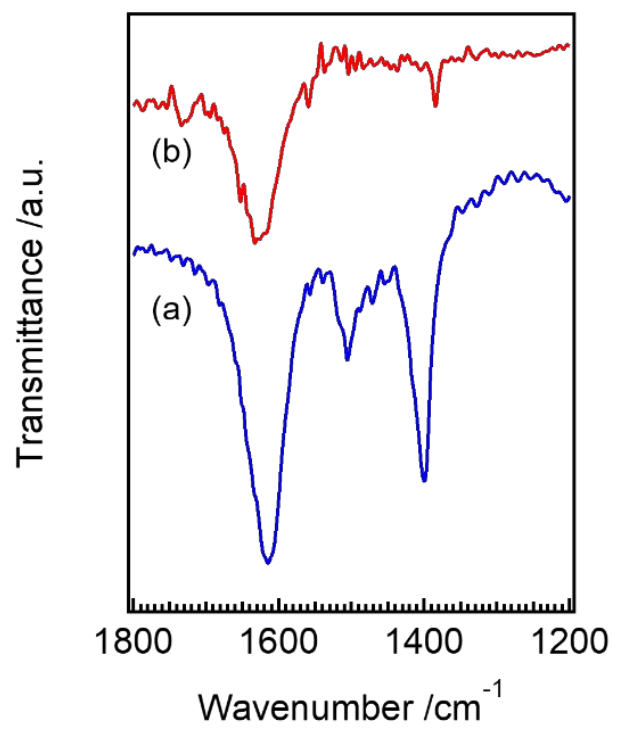

Figure S3. a TPD profiles of $1.8 \mathrm{~W}$ taken under the flow of $50 \mathrm{~mL} \mathrm{~min}^{-1}$ of $10 \% \mathrm{O}_{2} / \mathrm{He}$. b IR spectra of $1.8 \mathrm{~W}$ in the range of $1200 \sim 1800 \mathrm{~cm}^{-1}$ (a) before and (b) after the calcination at $400{ }^{\circ} \mathrm{C}$ for $2 \mathrm{~h}$ under air atmosphere. 
a

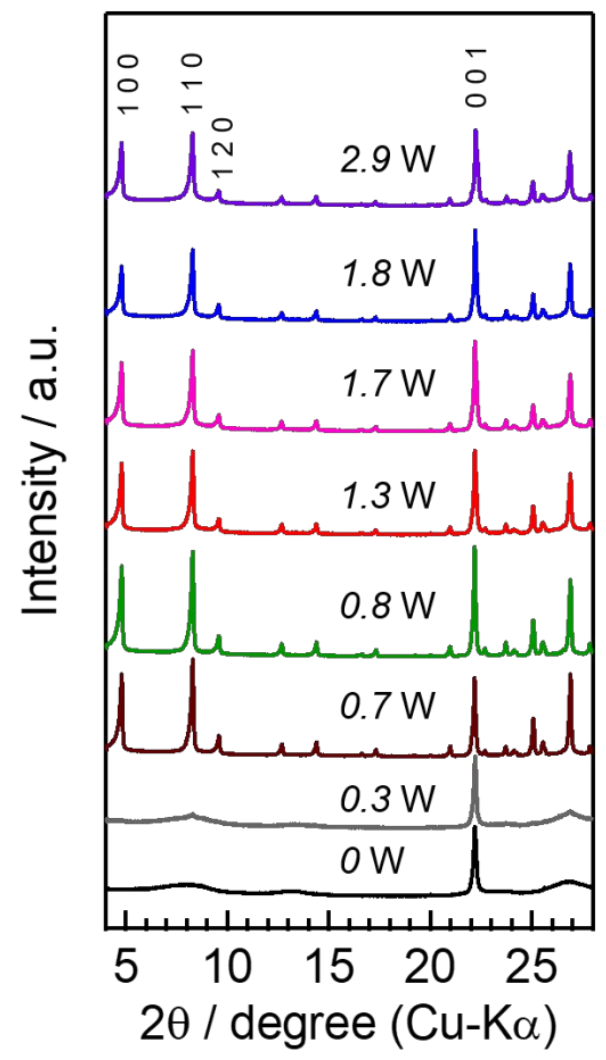

b

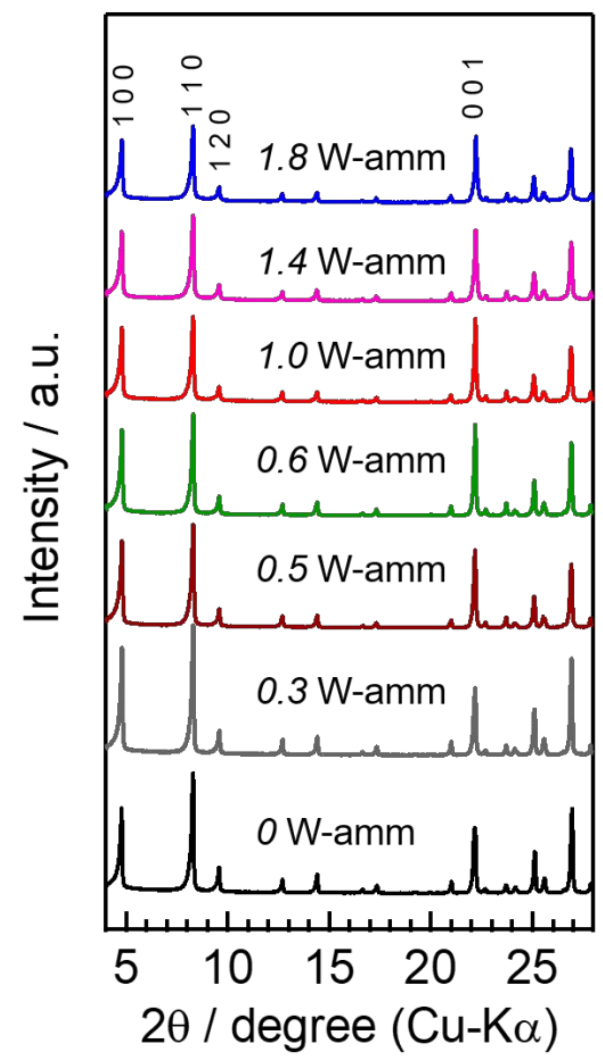

Figure S4. XRD patterns for $\mathbf{a} x \mathrm{~W}$ and $\mathbf{b} x \mathrm{~W}$-amm after air calcination at $400{ }^{\circ} \mathrm{C}$ for $2 \mathrm{~h}$. 

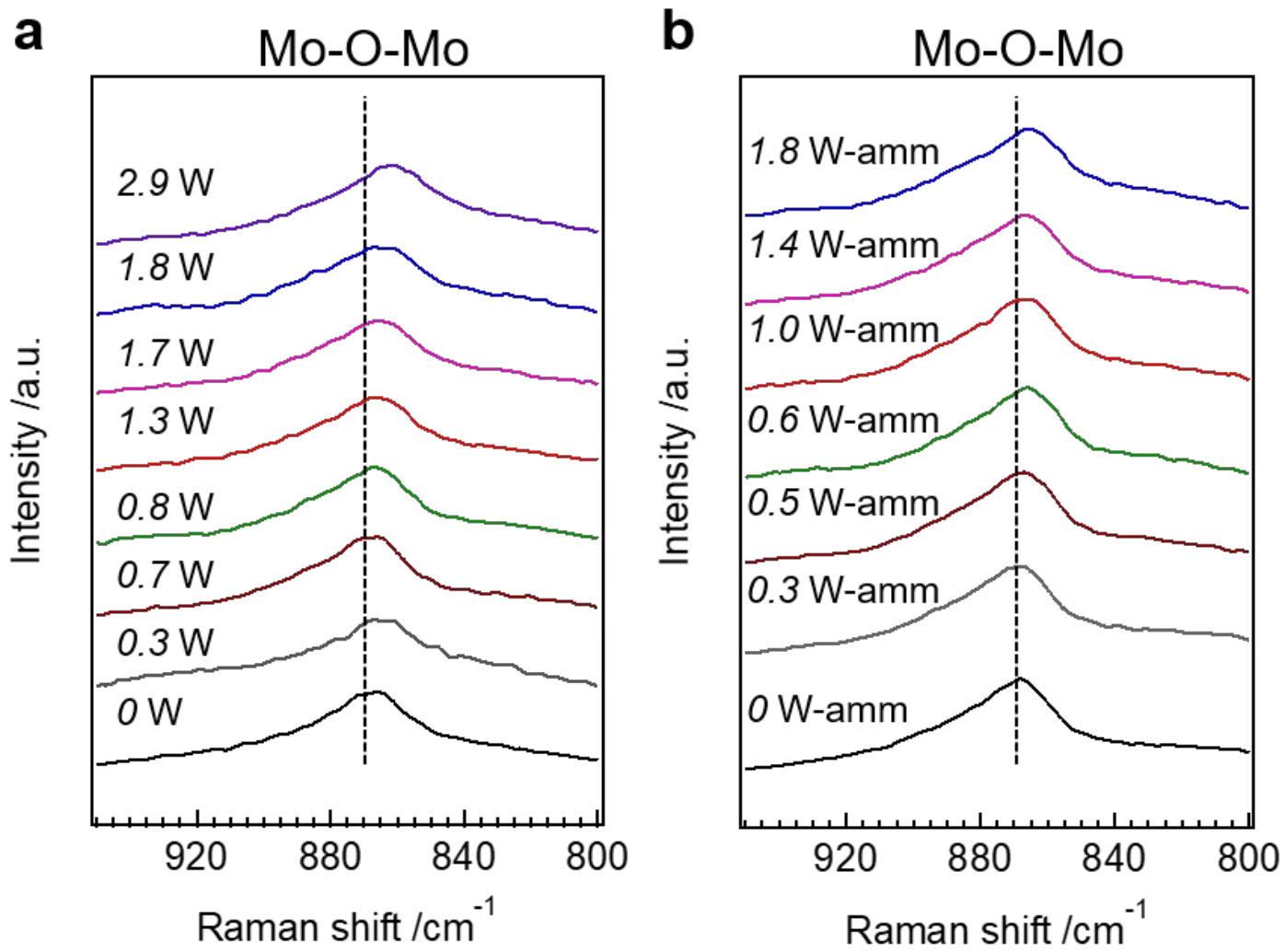

Figure S5. Raman spectra of $\mathbf{a} x \mathrm{~W}$ and $\mathbf{b} x \mathrm{~W}$-amm. 

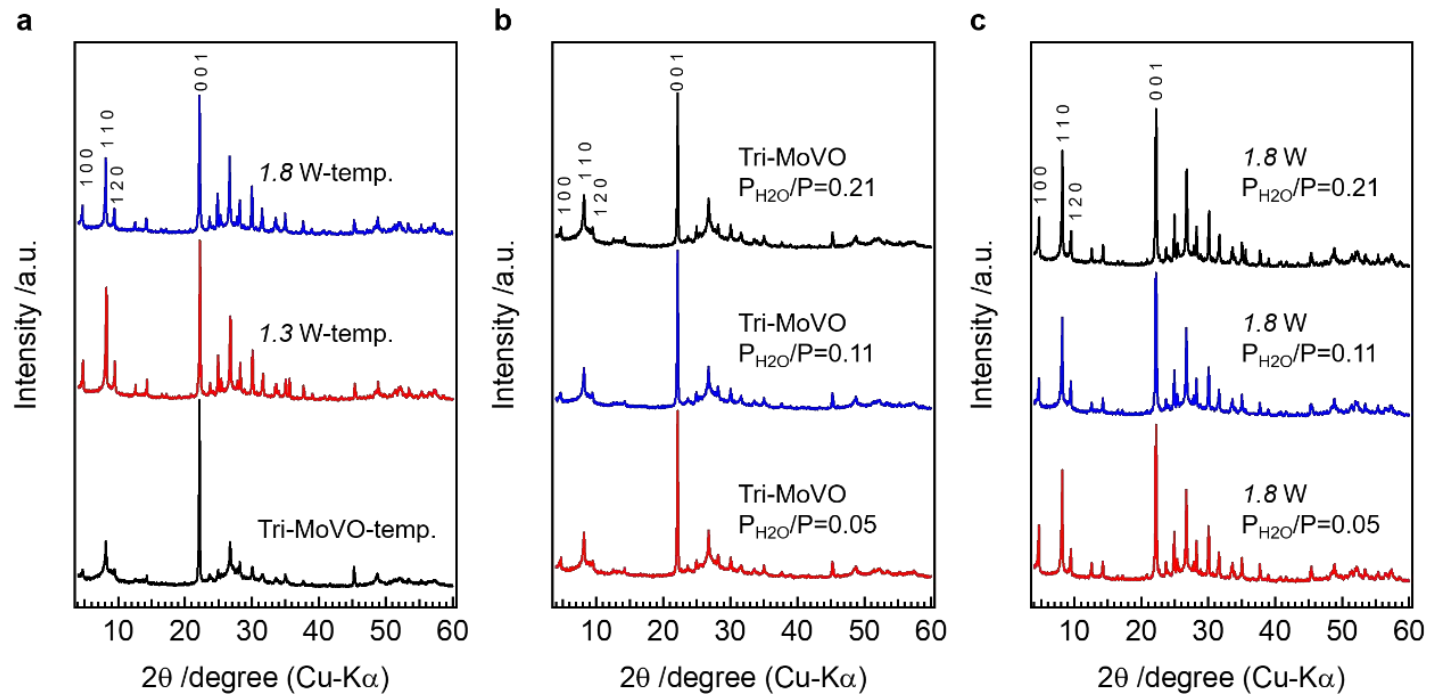

Figure S6. a XRD patterns for Tri-MoVO, $1.3 \mathrm{~W}$ and $1.8 \mathrm{~W}$ after reaction with varying reaction temperatures. b XRD patterns for Tri-MoVO after long-term reaction at various water pressures. $\mathrm{c}$ XRD patterns for $1.8 \mathrm{~W}$ after long-term reaction at various water pressures. 


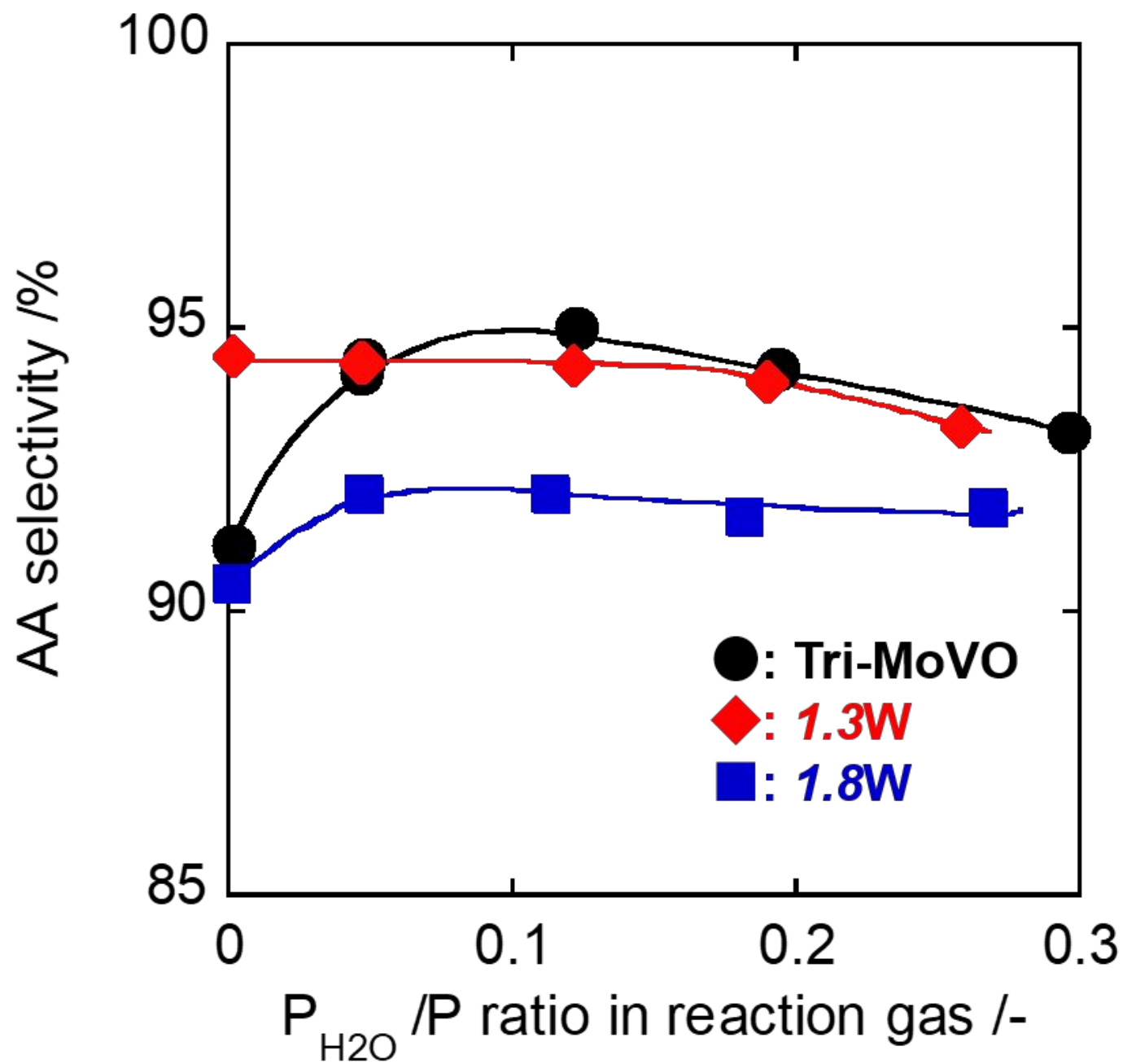

Figure S7. AA selectivity change as a function of water pressure in the reaction gas feed $\left(\mathrm{P}_{\mathrm{H} 2 \mathrm{O}} / \mathrm{P}\right)$ over Tri-MoVO, $1.3 \mathrm{~W}$ and $1.8 \mathrm{~W}$. Reaction conditions: catalyst amount, $0.15 \mathrm{~g}$; reaction temperature, $250{ }^{\circ} \mathrm{C}$; reaction gas, $\mathrm{ACR} / \mathrm{O}_{2} /\left(\mathrm{N}_{2}+\mathrm{He}\right) / \mathrm{H}_{2} \mathrm{O}=$ 1.3/4.0/45.0 58.0/0 13.0 $\mathrm{mL} \mathrm{min}^{-1}$ (total: $63.2 \mathrm{~mL} \mathrm{~min}^{-1}$ ). 

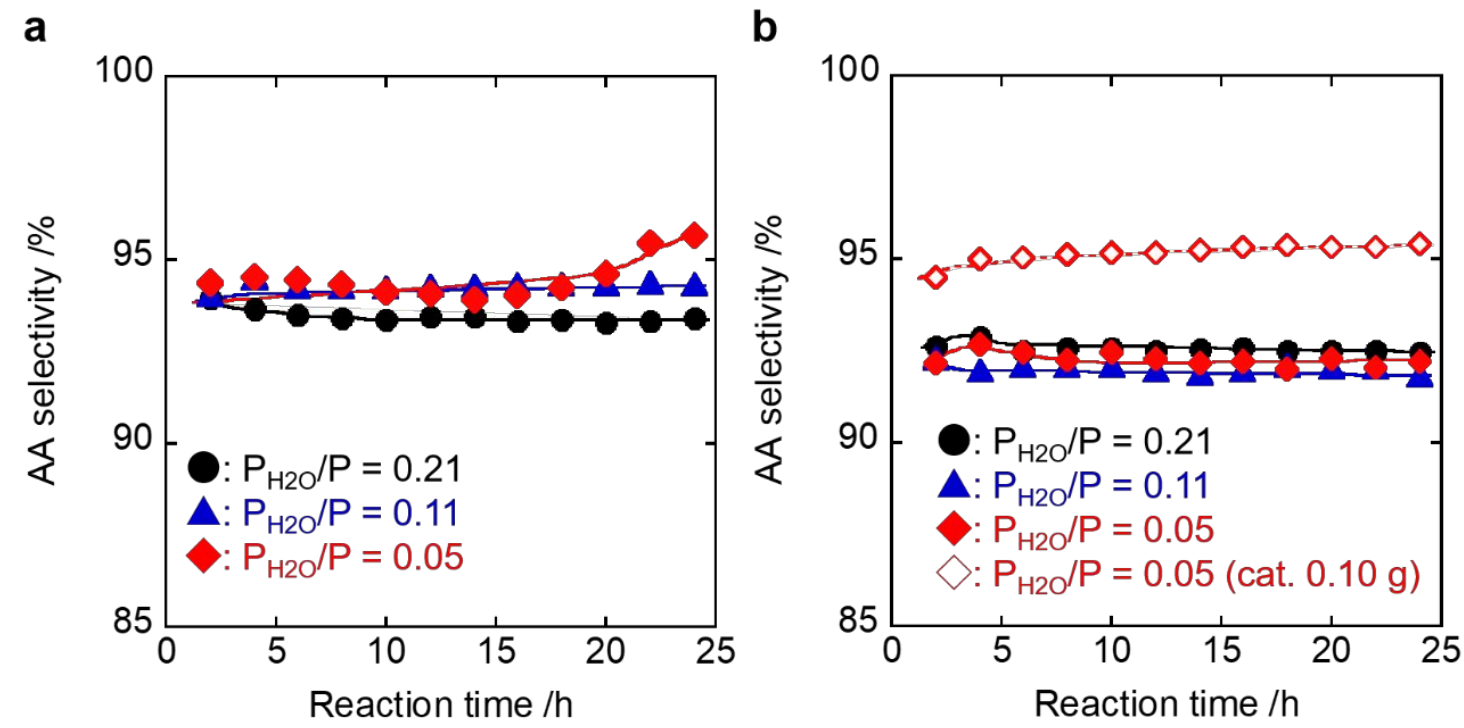

Figure S8. AA selectivity change as a function of reaction time at various water pressure conditions $\left(\mathrm{P}_{\mathrm{H} 2 \mathrm{O}} / \mathrm{P}\right)$ over a Tri-MoVO and b $1.8 \mathrm{~W}$. Reaction conditions: catalyst amount, $0.15 \mathrm{~g}$ without annotation; reaction temperature, $250{ }^{\circ} \mathrm{C}$; reaction gas, $\mathrm{ACR} / \mathrm{O}_{2} /\left(\mathrm{N}_{2}+\mathrm{He}\right) / \mathrm{H}_{2} \mathrm{O}=1.3 / 4.0 / 45.0 \sim 58.0 / 0 \sim 13.0 \mathrm{~mL} \mathrm{~min}^{-1}$ (total: $\left.63.2 \mathrm{~mL} \mathrm{~min}{ }^{-1}\right)$. 


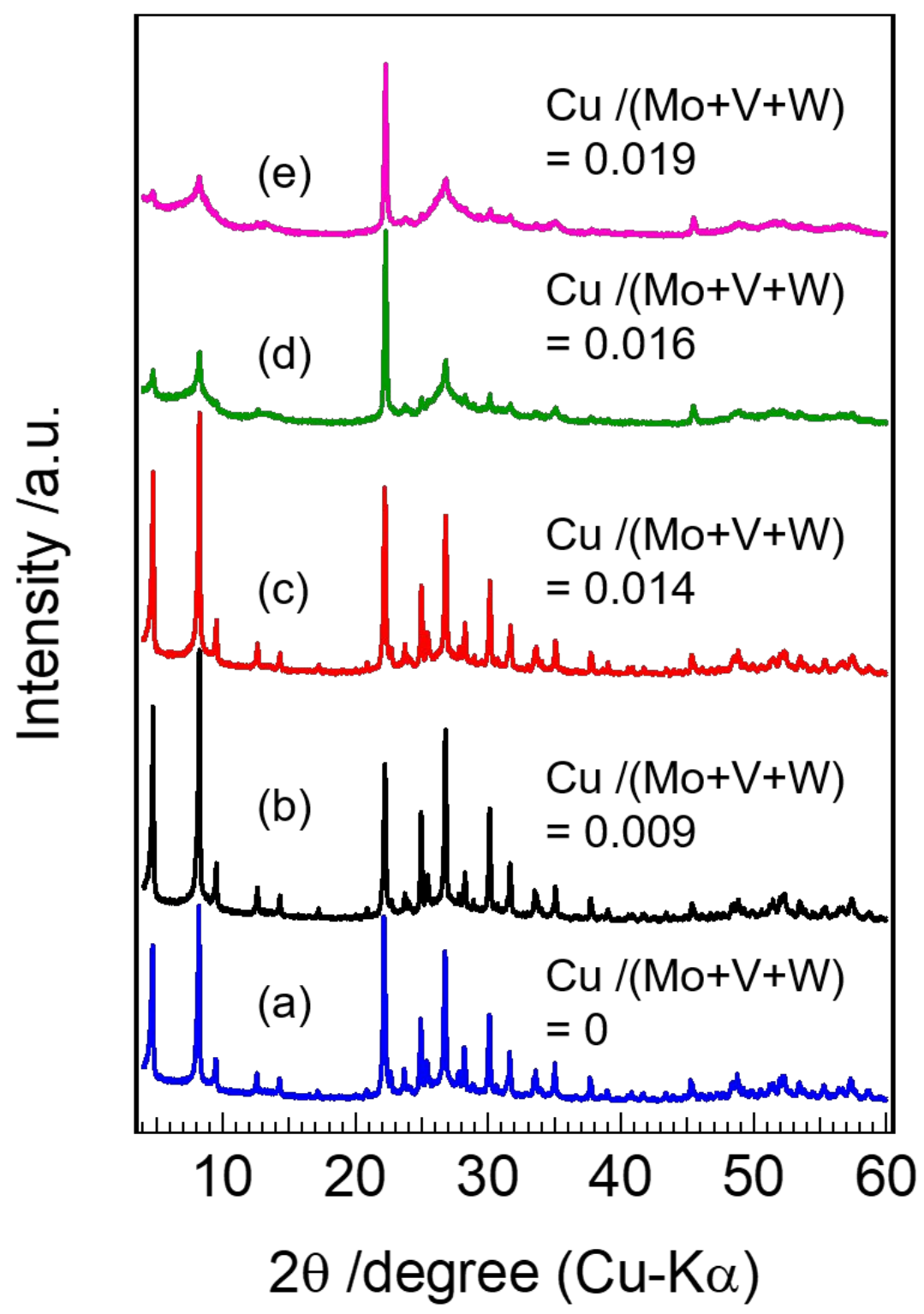

Figure S9. XRD patterns for Mo-V-W-Cu mixed oxides. Preparative W/(Mo+V) molar ratio was set to be 0.10 . Preparative $\mathrm{Cu} /(\mathrm{Mo}+\mathrm{V})$ molar ratios were (a) 0 , (b) 0.01 , (c) 0.02 , (d) 0.03 and (e) 0.05 . 


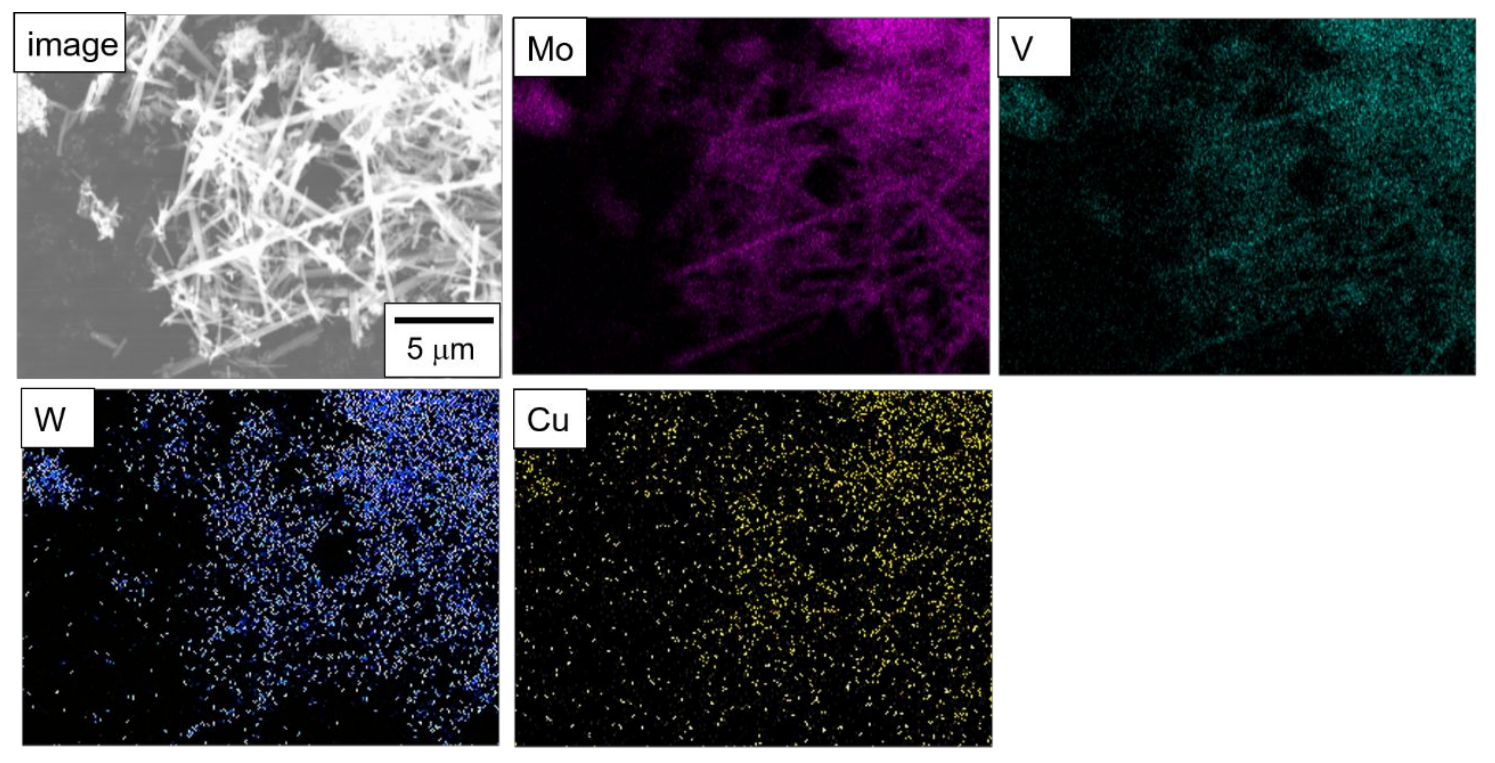

Figure S10. SEM mapping images of $2.4 \mathrm{~W} 0.4 \mathrm{Cu}$. 


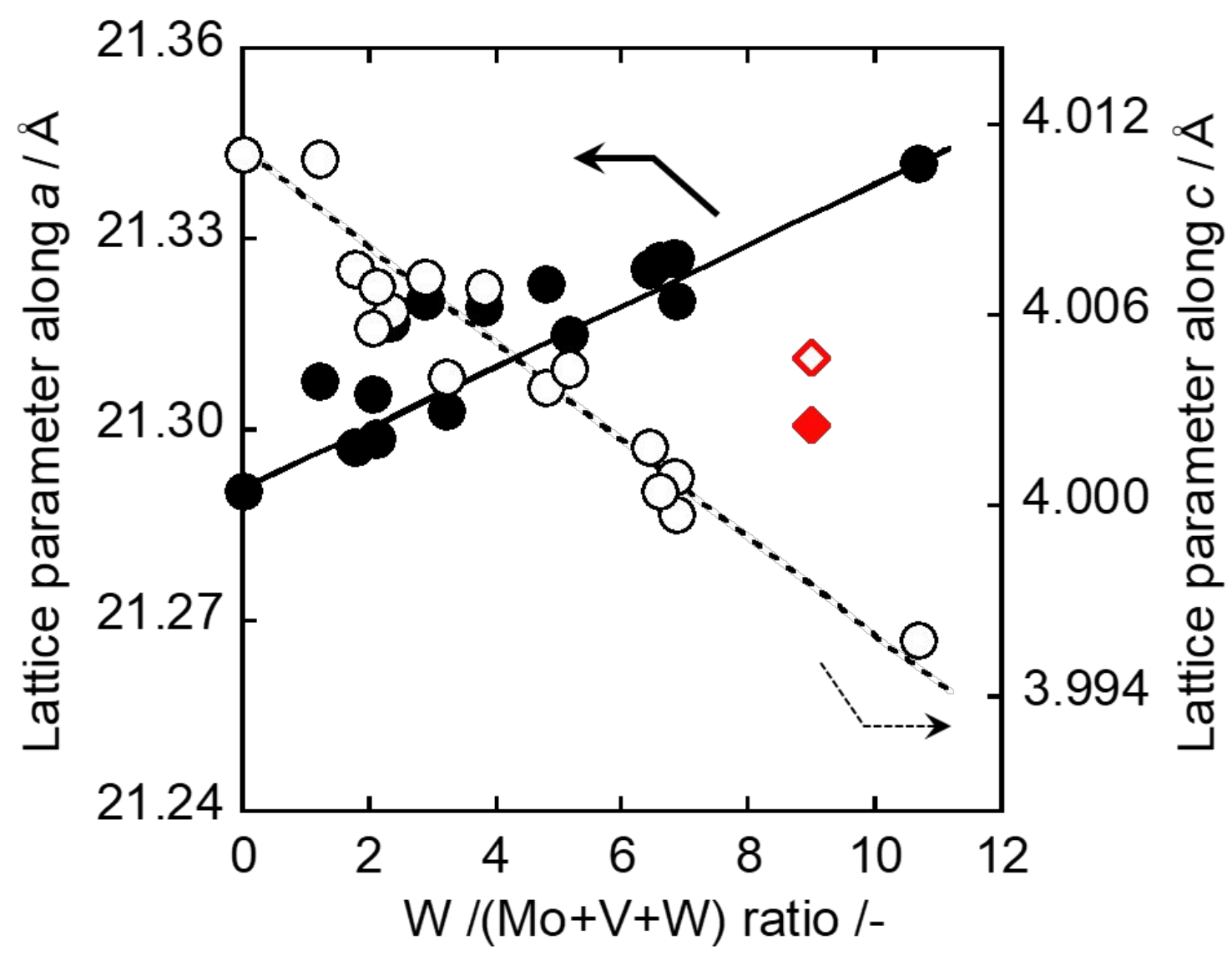

Figure S11. Lattice parameter changes as a function of $\mathrm{W} /(\mathrm{Mo}+\mathrm{V}+\mathrm{W})$ ratio. Lattice parameters of $x \mathrm{~W}-(\mathrm{amm})$ are shown as circles. Lattice parameters of $2.4 \mathrm{~W} 0.4 \mathrm{Cu}$ are represented as diamonds. Closed symbols, lattice parameter along $a$ axis; open symbols, lattice parameter along $c$ axis. 


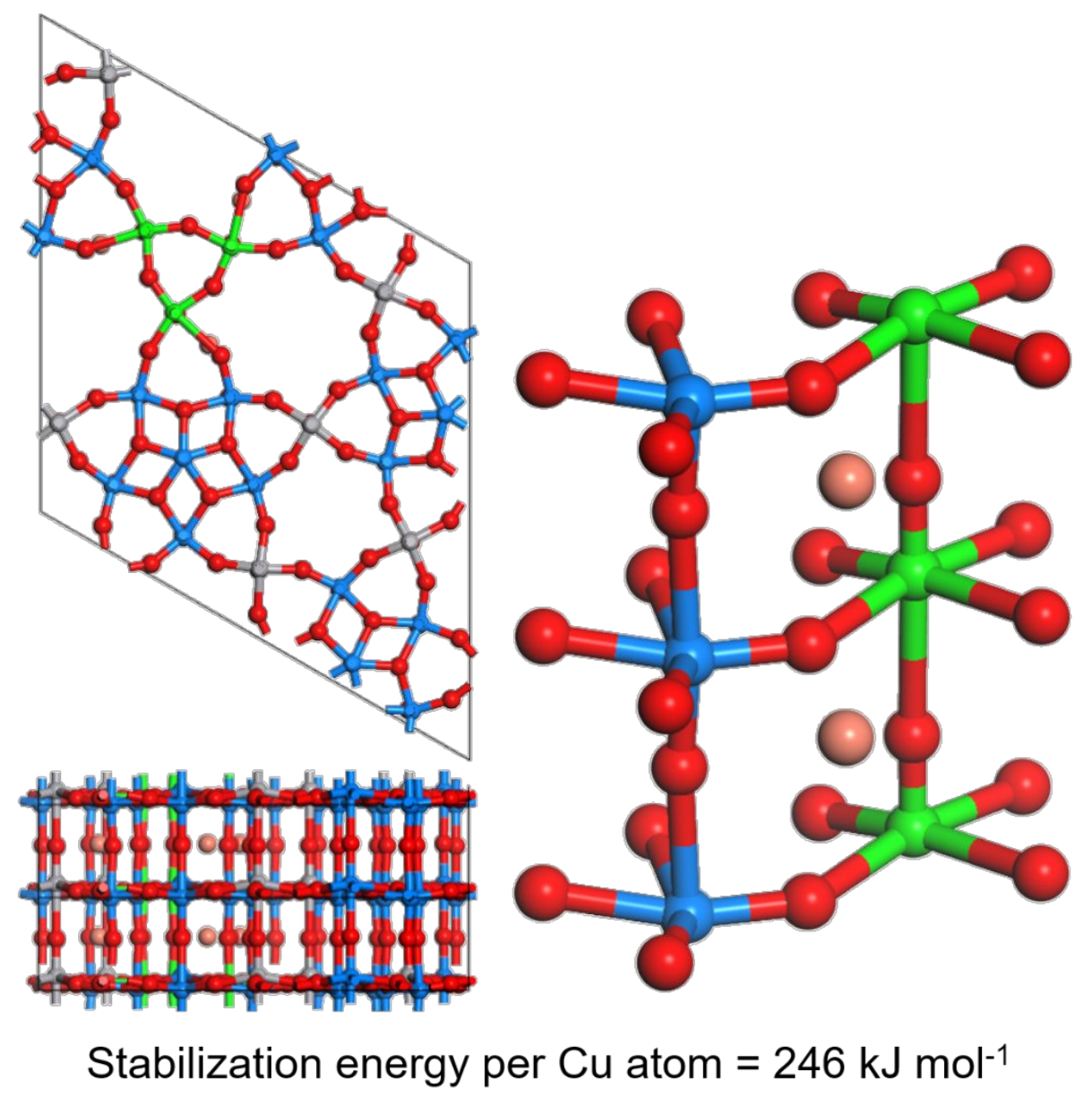

Figure S12. Structural model of $2.4 \mathrm{~W} 0.4 \mathrm{Cu}$ obtained after geometry optimization. Blue, mixture of Mo and $\mathrm{W}$; gray, $\mathrm{V}$; dark red, $\mathrm{Cu}$; light green, mixture of Mo and $\mathrm{V}$; red, $\mathrm{O}$. Local structure around $\mathrm{Cu}$ is shown at right. 


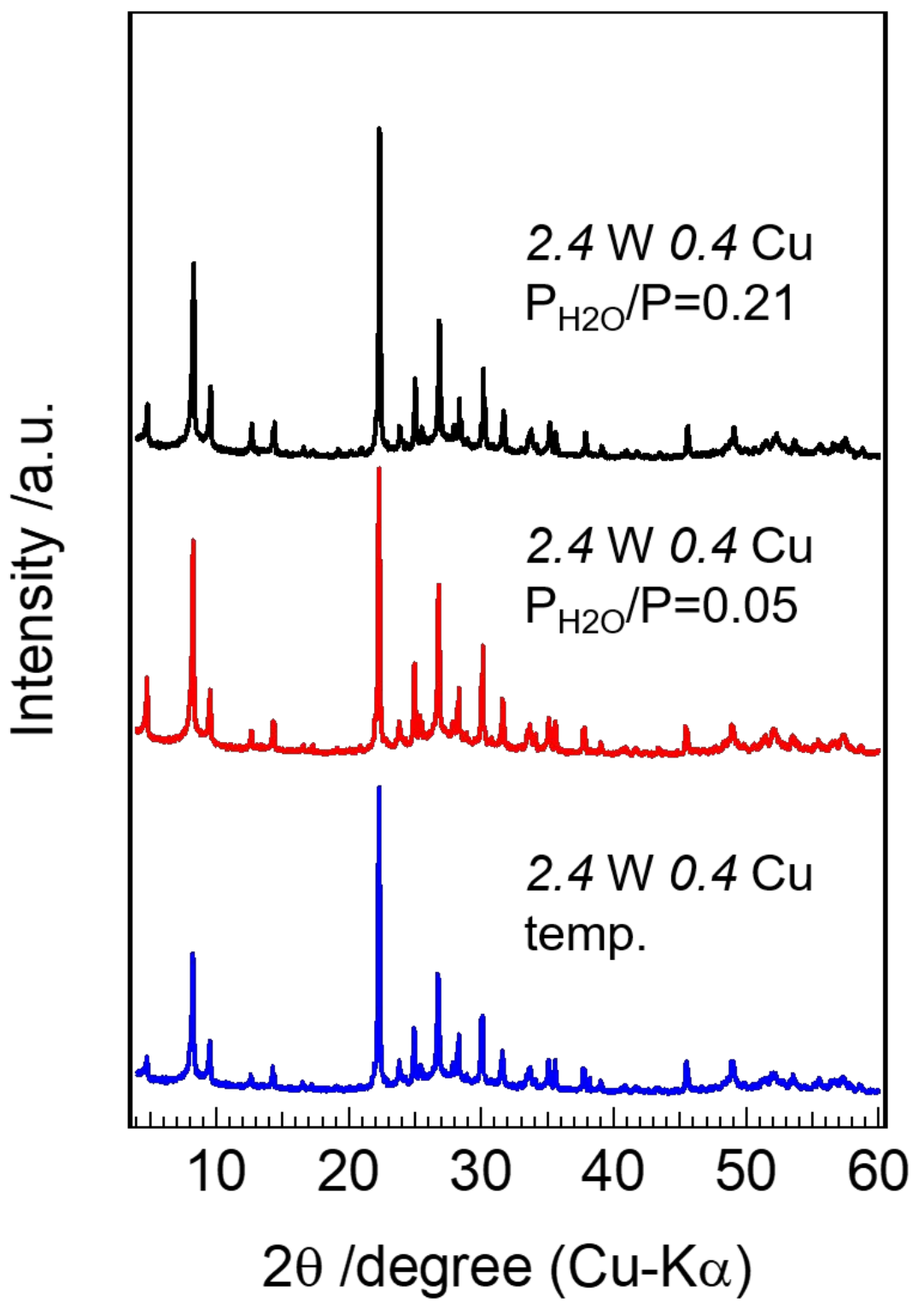

Figure S13. XRD patterns for $2.4 \mathrm{~W} 0.4 \mathrm{Cu}$ after the selective oxidation of ACR. 

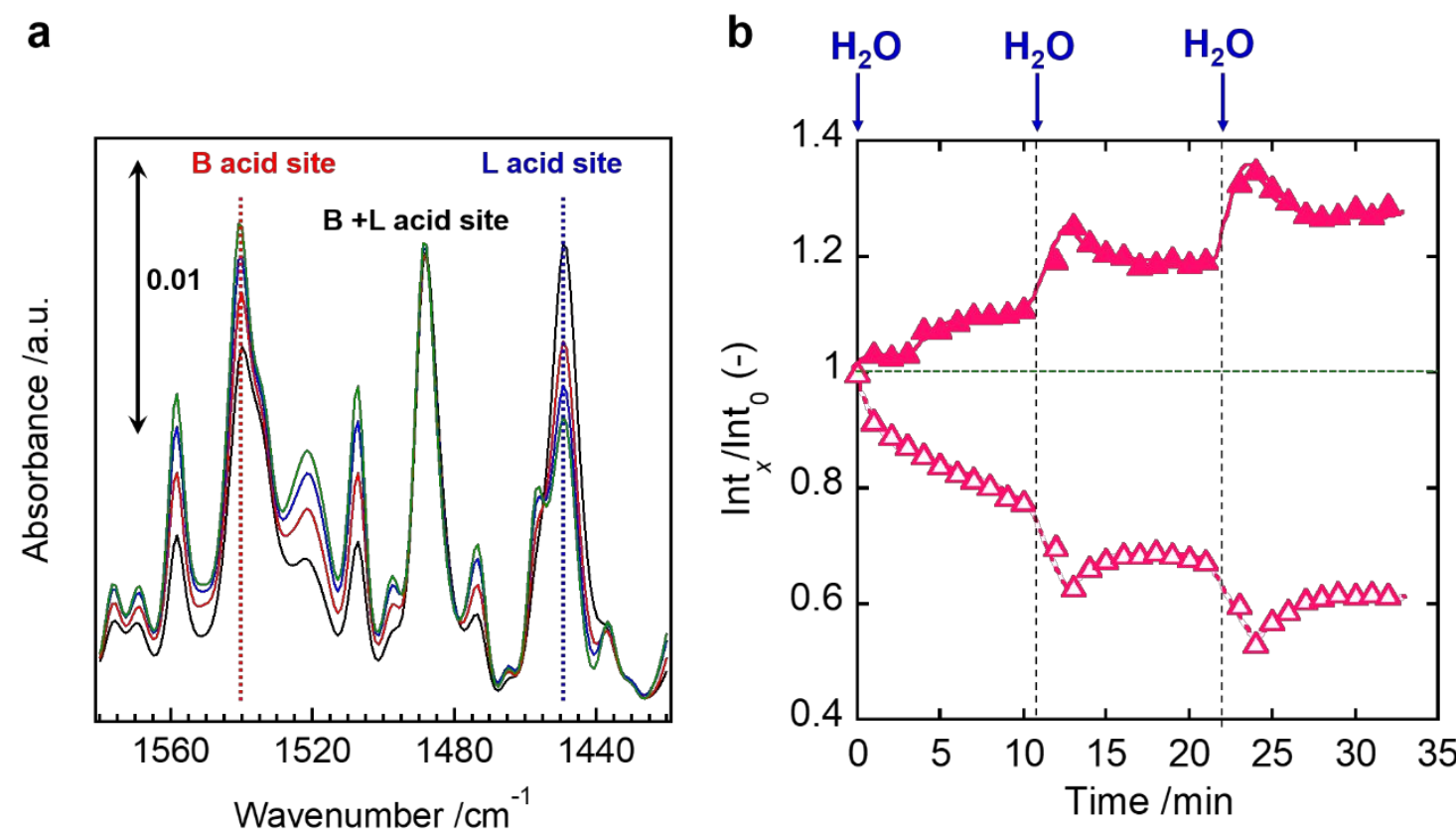

Figure S14. a IR spectra of pyridine adsorbed on $2.4 \mathrm{~W} 0.4 \mathrm{Cu}$ followed by the introduction of water $(0.03 \mathrm{~mL}$ as gas volume $)$ at three $10 \mathrm{~min}$ intervals at $250{ }^{\circ} \mathrm{C}$. Spectra obtained after 10 min from the water introduction are shown. Black, before water addition; red, after first water introduction; blue, after second water introduction; green, after third water introduction. b Changes of IR band intensities, $\mathrm{Int}_{x} / \mathrm{Int}_{0}$ (intensity at $x \mathrm{~min} /$ intensity at 0 min) derived from Brønsted acid sites (closed symbols) and Lewis acid sites (open symbols) as a function of measuring time $(x)$. Water was introduced at 10 min intervals. 


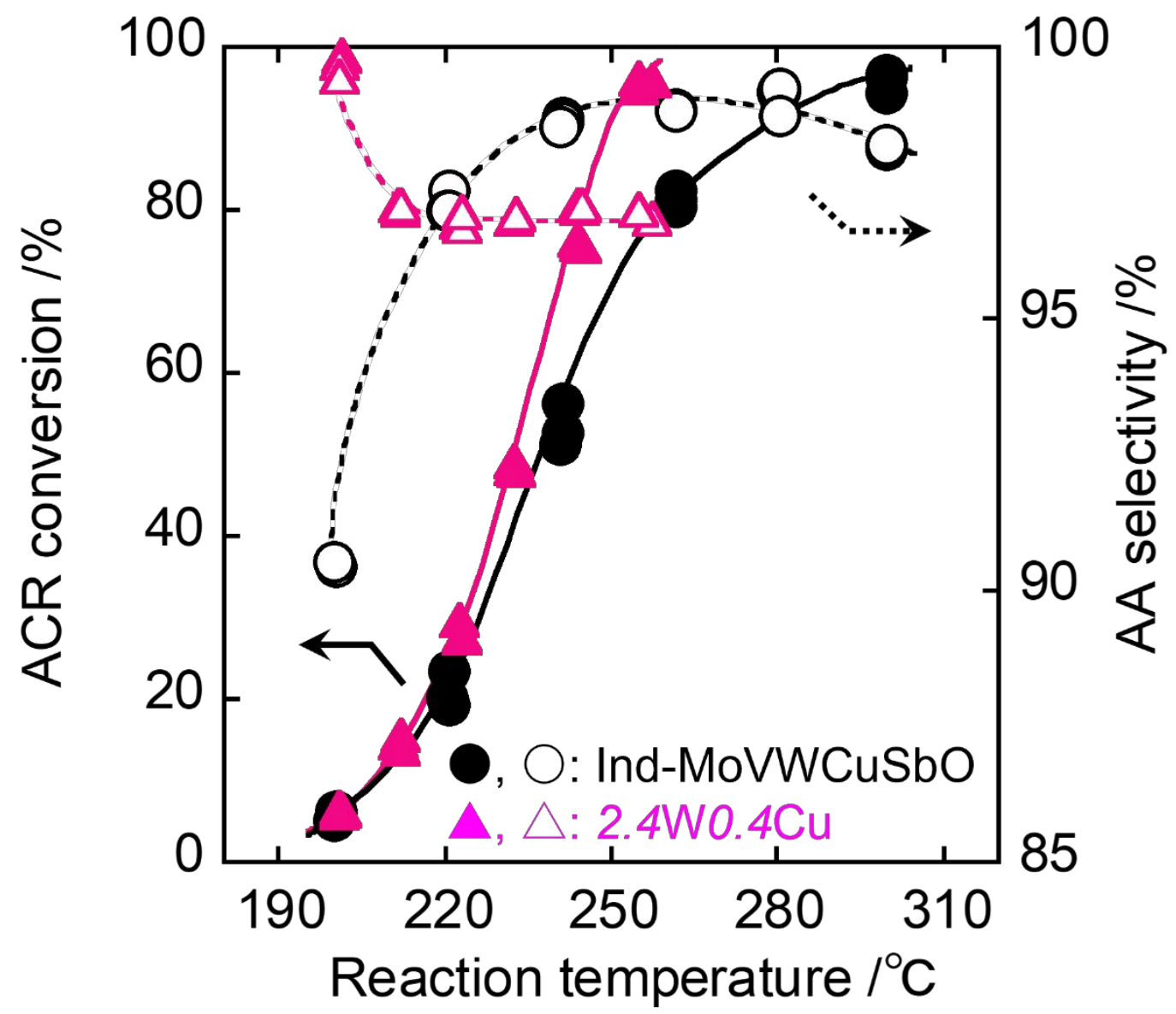

Figure S15. ACR conversion (closed symbols) and AA selectivity (open symbols) changes as a function of reaction temperature over Ind-MoVWCuSbO. Results of 2.4W0.4Cu was also shown as comparison. Reaction conditions: catalyst amount, $0.50 \mathrm{~g}$ (Ind-MoVWCuSbO) and $0.15 \mathrm{~g}(2.4 \mathrm{~W} 0.4 \mathrm{Cu})$; reaction temperature, $200 \sim 300{ }^{\circ} \mathrm{C}$; reaction gas, $\mathrm{ACR} / \mathrm{O}_{2} /\left(\mathrm{N}_{2}+\mathrm{He}\right) / \mathrm{H}_{2} \mathrm{O}=1.3 / 4.0 / 31.8 / 13.0 \mathrm{~mL} \mathrm{m^{-1 }}$ (total: $50.1 \mathrm{~mL} \mathrm{~min}^{-}$ $\left.{ }^{1}\right)$. 
Table S1. Catalytic activity of the industrial catalysts

\begin{tabular}{ccccc}
\hline Catalyst & Temp. ${ }^{\mathrm{a}} /{ }^{\circ} \mathrm{C}$ & ACR conv. $\%$ & AA sel. $/ \%$ & Reference \\
\hline $\mathrm{Mo}_{12} \mathrm{~V}_{3.47} \mathrm{~W}_{1.39} \mathrm{O}_{\mathrm{x}}$ & 255 & 99 & 92.4 & \\
$\mathrm{Mo}_{12} \mathrm{~V}_{3} \mathrm{~W}_{1.2} \mathrm{Cu}_{1.6} \mathrm{O}_{\mathrm{x}}$ & 254 & 99 & 96.0 & {$[1]$} \\
$\mathrm{Mo}_{12} \mathrm{~V}_{3} \mathrm{~W}_{1.2} \mathrm{Cu}_{1.2} \mathrm{Sb}_{0.5} \mathrm{O}_{\mathrm{x}}$ & 250 & 99.1 & 98.5 & {$[2]$} \\
$\mathrm{Mo}_{12} \mathrm{~V}_{4} \mathrm{~W}_{2.5} \mathrm{Cu}_{2} \mathrm{Sr}_{0.2} \mathrm{O}_{\mathrm{x}}$ & $250^{\mathrm{a}}$ & 99.3 & 94.0 & {$[3]$} \\
\hline
\end{tabular}

a Temperature of the reaction bath.

${ }^{\mathrm{b}}$ Reaction temperature in the catalyst layer was $305-308{ }^{\circ} \mathrm{C}$ 
Table S2. Amounts of cations in precursor solution and in solid, and results of $\mathrm{N}_{2}$ adsorption experiments

\begin{tabular}{|c|c|c|c|c|c|c|c|}
\hline \multirow[t]{2}{*}{ Catalyst } & \multicolumn{2}{|c|}{$\begin{array}{l}\text { Concentration of } \\
\text { cations in precursor } \\
\text { solution } / \mathrm{mmol} \mathrm{L}^{-1}\end{array}$} & \multicolumn{3}{|c|}{$\begin{array}{l}\text { Number of cations in Tri- } \\
\text { MoVO unit cell }\end{array}$} & \multirow{2}{*}{$\begin{array}{l}\text { External } \\
\text { surface area } \\
/ \mathrm{m}^{2} \mathrm{~g}^{-1}\end{array}$} & \multirow{2}{*}{$\begin{array}{l}\text { Micropore } \\
\text { volume } \\
\mathrm{m}^{2} \mathrm{~g}^{-1}\end{array}$} \\
\hline & $\mathrm{NH}_{4}^{+}$ & $\mathrm{EtNH}_{3}{ }^{+}$ & $\mathrm{EtNH}_{3}{ }^{+}$ & $\mathrm{NH}_{4}^{+}$ & Sum & & \\
\hline Tri-MoVO & 179 & 0 & 0 & 2.1 & 2.1 & 19.6 & 2.4 \\
\hline $0 \mathrm{~W}$ & 0 & 139 & 2.0 & 0 & 2.0 & 21.7 & 6.3 \\
\hline $0.3 \mathrm{~W}$ & 1 & 139 & 2.1 & 0 & 2.1 & 33.4 & 6.7 \\
\hline $0.7 \mathrm{~W}$ & 3 & 139 & 2.1 & 0.3 & 2.4 & 22.2 & 1.4 \\
\hline $0.8 \mathrm{~W}$ & 4 & 139 & 2.1 & 0.6 & 2.7 & 18.3 & 2.4 \\
\hline $1.3 \mathrm{~W}$ & 7 & 139 & 2.1 & 0.7 & 2.8 & 23.9 & 2.9 \\
\hline $1.7 \mathrm{~W}$ & 10 & 139 & 2.1 & 0.6 & 2.7 & 25.0 & 3.7 \\
\hline $1.8 \mathrm{~W}$ & 13 & 139 & 2.1 & 0.5 & 2.6 & 24.9 & 3.5 \\
\hline $2.9 \mathrm{~W}$ & 20 & 139 & 2.1 & 0.8 & 2.9 & 23.7 & 4.6 \\
\hline $0 \mathrm{~W}$-amm & 20 & 139 & 2.1 & 1.1 & 3.2 & 4.8 & 0.7 \\
\hline $0.3 \mathrm{~W}$-amm & 20 & 139 & 2.0 & 0.8 & 2.8 & 5.8 & 0.3 \\
\hline $0.5 \mathrm{~W}-\mathrm{amm}$ & 20 & 139 & 2.1 & 0.8 & 2.9 & 8.8 & 0.3 \\
\hline $0.6 \mathrm{~W}-\mathrm{amm}$ & 20 & 139 & 2.1 & 0.8 & 2.9 & 8.3 & 0.3 \\
\hline $1.0 \mathrm{~W}-\mathrm{amm}$ & 20 & 139 & 2.1 & 0.5 & 2.6 & 14.5 & 1.2 \\
\hline $1.4 \mathrm{~W}-\mathrm{amm}$ & 20 & 139 & 2.1 & 0.7 & 2.8 & 23.4 & 1.0 \\
\hline $1.8 \mathrm{~W}$-amm & 20 & 139 & 2.1 & 0.8 & 2.9 & 20.8 & 2.2 \\
\hline
\end{tabular}

a Determined by CHN elemental analysis.

${ }^{b}$ Measured by $\mathrm{N}_{2}$ adsorption at liq. $\mathrm{N}_{2}$ temperature and determined by $\alpha_{\mathrm{s}}$-plot method. 
Table S3. External surface areas and micropore volumes of catalysts after the selective oxidation of ACR

\begin{tabular}{ccc}
\hline Catalyst $^{\mathrm{a}}$ & External surface $\mathrm{area}^{\mathrm{b}} / \mathrm{m}^{2} \mathrm{~g}^{-1}$ & Micropore volume $^{\mathrm{b}} / 10^{-3} \mathrm{~cm}^{3} \mathrm{~g}^{-1}$ \\
\hline Tri-MoVO-temp. & 19.6 & 1.1 \\
$1.3 \mathrm{~W}$-temp. & 23.4 & 3.0 \\
$1.8 \mathrm{~W}$-temp. & 24.3 & 2.8 \\
Tri-MoVO & 18.8 & 1.0 \\
$\left(\mathrm{P} / \mathrm{P}_{\mathrm{H} 2 \mathrm{O}}=0.05\right)$ & 18.1 & 1.3 \\
Tri-MoVO & & \\
$\left(\mathrm{P} / \mathrm{P}_{\mathrm{H} 2 \mathrm{O}}=0.21\right)$ & 24.9 & 2.9 \\
$1.8 \mathrm{~W}$ & & 2.4 \\
$\left(\mathrm{P} / \mathrm{P}_{\mathrm{H} 2 \mathrm{O}}=0.05\right)$ & 25.1 & \\
$1.8 \mathrm{~W}$ & & \\
$\left(\mathrm{P} / \mathrm{P}_{\mathrm{H} 2 \mathrm{O}}=0.21\right)$ & & \\
\hline
\end{tabular}

a Catalysts after the reaction with varying reaction temperature are denoted as -temp. Catalysts after the reaction with varying water pressure are shown with the water pressure used for the reaction.

${ }^{b}$ Measured by $\mathrm{N}_{2}$ adsorption at liq. $\mathrm{N}_{2}$ temperature and determined by $\alpha_{\mathrm{s}}$-plot method. 
Table S4. Physicochemical properties of $1.8 \mathrm{~W} 0.4 \mathrm{Cu}$ catalysts

\begin{tabular}{llllll}
\hline \multirow{2}{*}{ Catalyst } & $\begin{array}{l}\text { Elemental } \\
\text { composition } \\
(\mathrm{Mo} / \mathrm{V} / \mathrm{W} / \mathrm{Cu})\end{array}$ & \multicolumn{2}{c}{ Lattice parameter $/ \mathrm{nm}$} & $\begin{array}{l}\text { External surface } \\
\mathrm{area}^{\mathrm{b}} \\
/ \mathrm{m}^{2} \mathrm{~g}^{-1}\end{array}$ & $\begin{array}{l}\text { Micropore } \\
\text { volume }^{\mathrm{b}}\end{array}$ \\
\cline { 3 - 6 } $\mathrm{cm}^{3} \mathrm{~g}^{-1}$
\end{tabular}

a Determined by ICP.

${ }^{b}$ Measured by $\mathrm{N}_{2}$ adsorption at liq. $\mathrm{N}_{2}$ temperature and determined by $\alpha_{\mathrm{s}}$-plot method. External surface areas and micropore volumes of catalysts after ACR oxidation are shown in parenthesis. 
Table S5. Refined parameters and agreement factors for Rietveld refinement of $1.8 \mathrm{~W}$ after air calcination

\begin{tabular}{ll}
\hline & $1.8 \mathrm{~W}$ \\
Crystal system & Trigonal \\
Space group & $\mathrm{P} 3$ \\
$a / \AA$ & $21.3203(8)$ \\
$c / \AA$ & $3.9997(4)$ \\
$\alpha=\beta / \mathrm{deg}$ & 90 \\
$\gamma / \mathrm{deg}$ & 120 \\
$\mathrm{~V} / \AA^{3}$ & 1574.57 \\
Agreement factors & \\
$\mathrm{R}_{\mathrm{wp}} / \%$ & 9.15 \\
$\mathrm{R}_{\mathrm{wp}}($ without background) $/ \%$ & 26.77 \\
$\mathrm{R}_{\mathrm{p}} / \%$ & 6.66 \\
Pattern parameter & Pseudo Voigt \\
Peak shape function & $\mathrm{U}=0.34773, \mathrm{~V}=-0.21691, \mathrm{~W}=0.04722$ \\
FWHM & $\mathrm{N}_{\mathrm{A}}=1.05252, \mathrm{~N}_{\mathrm{B}}=-0.01313$ \\
Profile parameter & \\
Line shift & Bragg-Brentano \\
Instrument geometry & -0.15905 \\
Zero point & 0.10290 \\
Shift $\# 1$ & 0.11764 \\
Shift $\# 2$ & Berar-Baldinozzi \\
Correction method & $\mathrm{P}_{1}=-1.53239, \mathrm{P}_{2}=-0.42869, \mathrm{P}_{3}=2.93707, \mathrm{P}_{4}=0.83003$ \\
Parameter &
\end{tabular}


Table S6. Atomic coordinates and occupancy of the framework of $1.8 \mathrm{~W}$

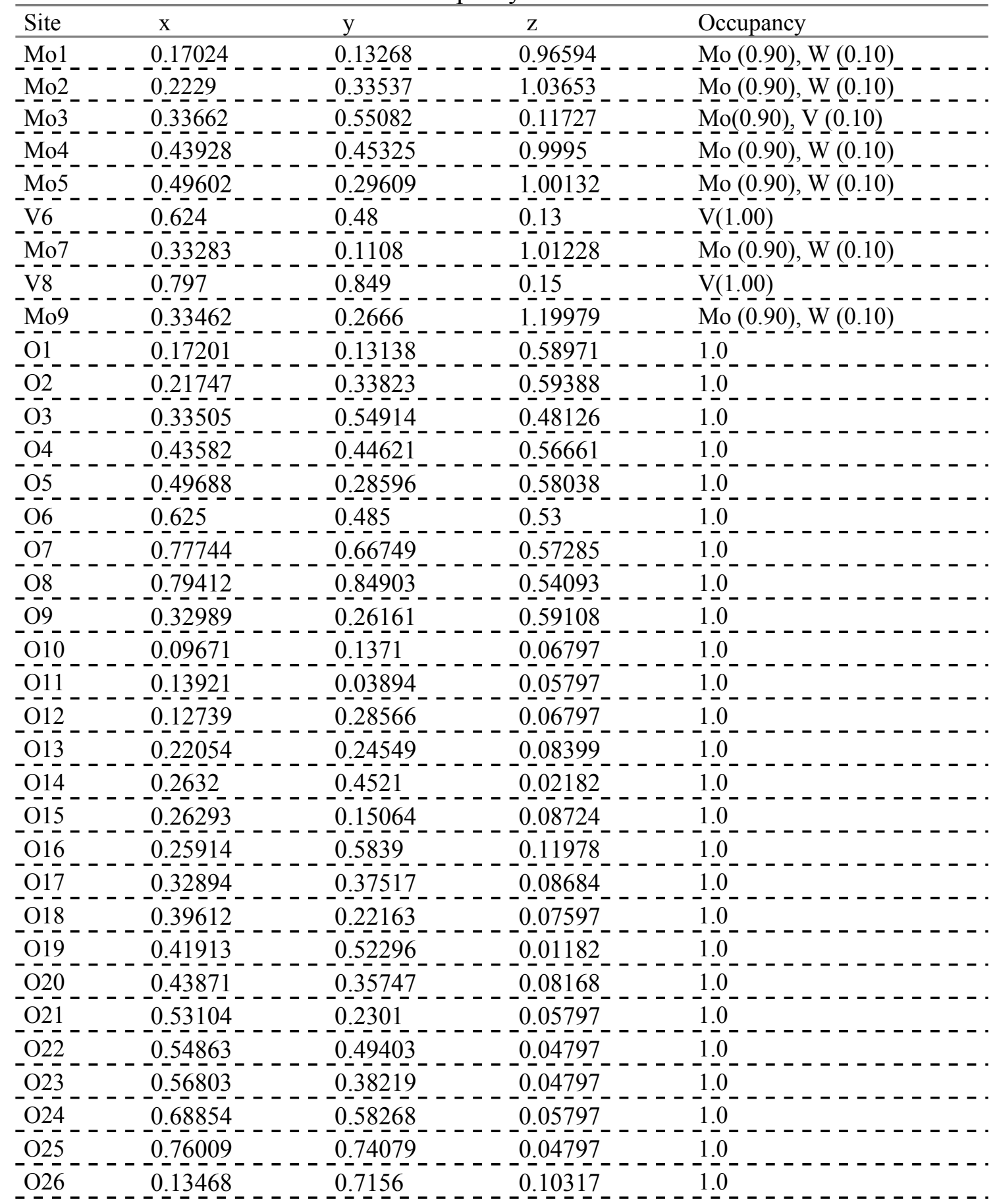


Table S7. Refined parameters and agreement factors for Rietveld refinement of $2.4 \mathrm{~W} 0.4 \mathrm{Cu}$ after the air calcination

\begin{tabular}{ll}
\hline & $2.4 \mathrm{~W} 0.4 \mathrm{Cu}$ \\
Crystal system & Trigonal \\
Space group & $\mathrm{P} 3$ \\
$a / \AA$ & $21.3006(9)$ \\
$c / \AA$ & $4.0048(8)$ \\
$\alpha=\beta / \mathrm{deg}$ & 90 \\
$\gamma / \mathrm{deg}$ & 120 \\
$\mathrm{~V} / \AA^{3}$ & 1572.02 \\
Agreement factors & \\
$\mathrm{R}_{\mathrm{wp}} / \%$ & 8.69 \\
$\mathrm{R}_{\mathrm{wp}}($ without background) $/ \%$ & 22.23 \\
$\mathrm{R}_{\mathrm{p}} / \%$ & 6.63 \\
Pattern parameter & Pseudo Voigt \\
Peak shape function & $\mathrm{U}=0.25690, \mathrm{~V}=-0.08625, \mathrm{~W}=0.03133$ \\
FWHM & $\mathrm{N}_{\mathrm{A}}=1.02476, \mathrm{~N}_{\mathrm{B}}=-0.00619$ \\
Profile parameter & \\
Line shift & Bragg-Brentano \\
Instrument geometry & -0.26568 \\
Zero point & 0.19543 \\
Shift \#1 & 0.25221 \\
Shift $\# 2$ & Berar-Baldinozzi \\
Correction method & $\mathrm{P}_{1}=-0.09045, \mathrm{P}_{2}=0.05411, \mathrm{P}_{3}=0.03233, \mathrm{P}_{4}=-0.14216$ \\
Parameter &
\end{tabular}


Table S8. Atomic coordinates and occupancy for the framework of $2.4 \mathrm{~W} 0.4 \mathrm{Cu}$

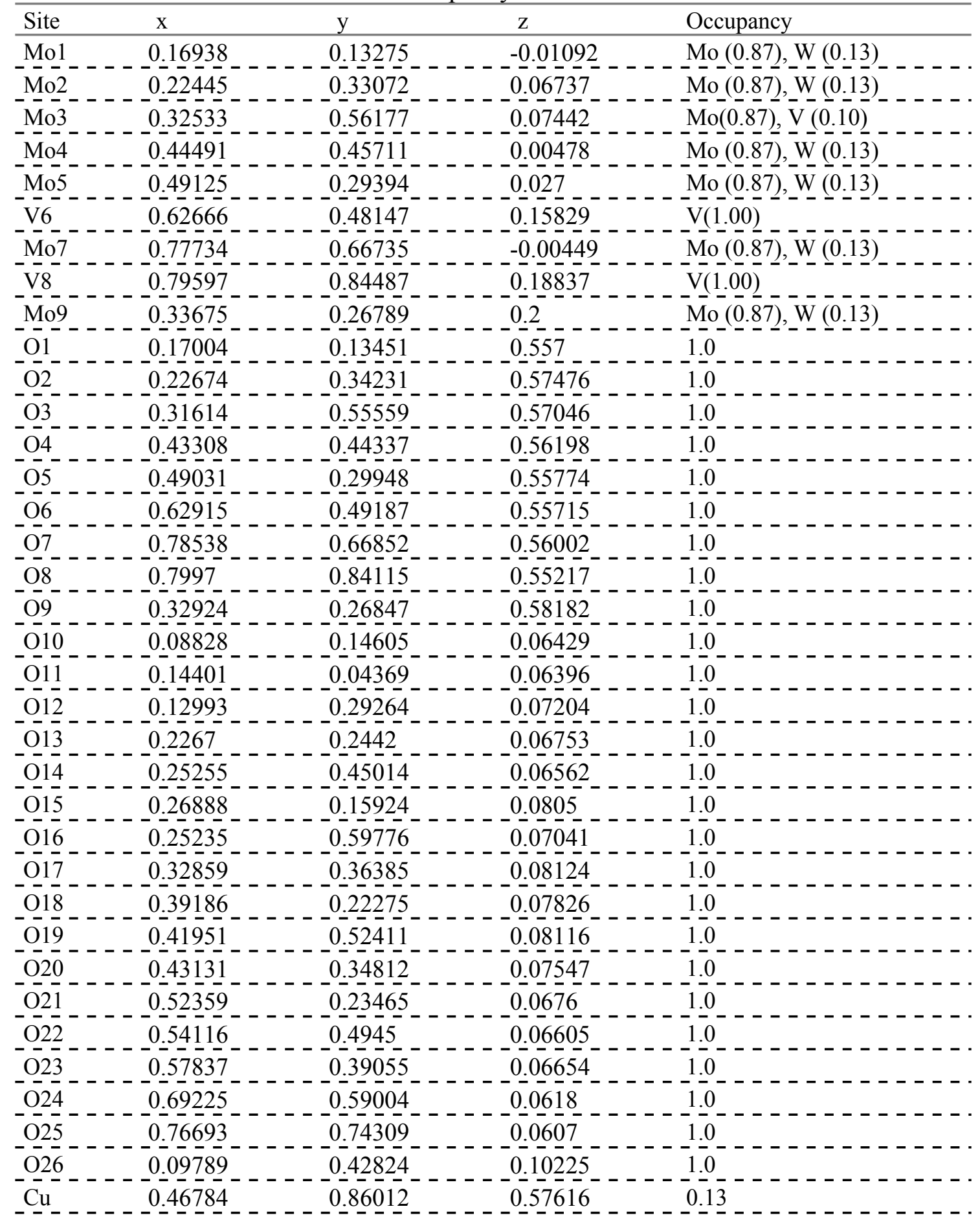




\section{Reference}

[1] Hibst, H; Tenten, A.; Marosi, L. Multimetal Oxides. U.S. Patent US 5,807,531.

[2] Sugi, H; Sakai, F.; Wada, K.; Shiraishi, K.; Kojima, T.; Umejima, A.; Seo, Y.

Catalysts and Process for the Preparation Thereof. U.S. Patent US 5,959,143.

[3] Kawajiri, T.; Tanimoto, M.; Nakamura, D. Process for Production of Acrylic Acid.

Euro. Patent EP 0,792,866,A1. 